Supporting Information

\title{
The Comparison Investigation on the Adsorption Affinity of DNA Molecules to Gold Surface based on the Kinetic and Thermodynamic Analysis of 4- Nitrophenol Reduction
}

Ting Zhou*, Chenqi Zhang, Hong Wang, Xuefeng Kang, Zhiqing Zhang, * Fang Wang, Xiufeng Wang, Guodong Zhang

Department of Chemistry, College of Science, China University of Petroleum (East China), Qingdao, 266580, P. R. China
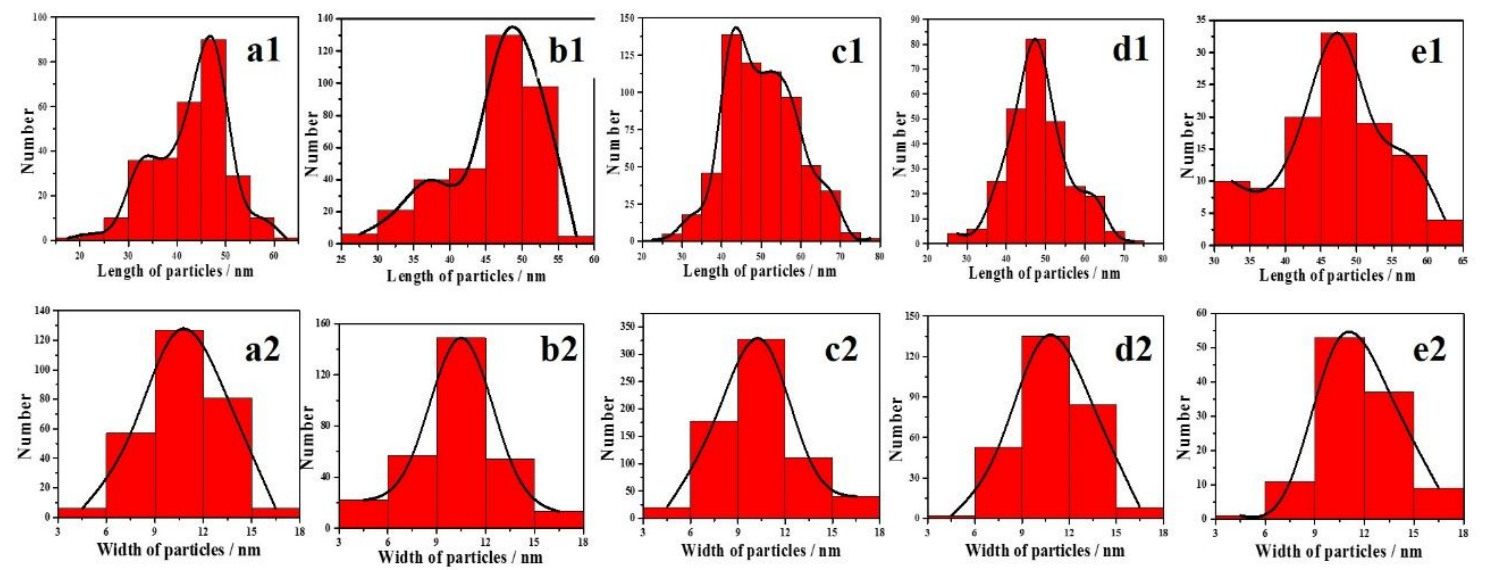

Figure S1. Length and width distribution with Gaussian fitting (black lines) of AuNRs and nucleobases modified AuNPs. (a1, a2) AuNRs, (b1, b2) A-AuNRs, (c1, c2) G-AuNRs, (d1, d2) CAuNRs, (e1, e2) T -AuNRs. 

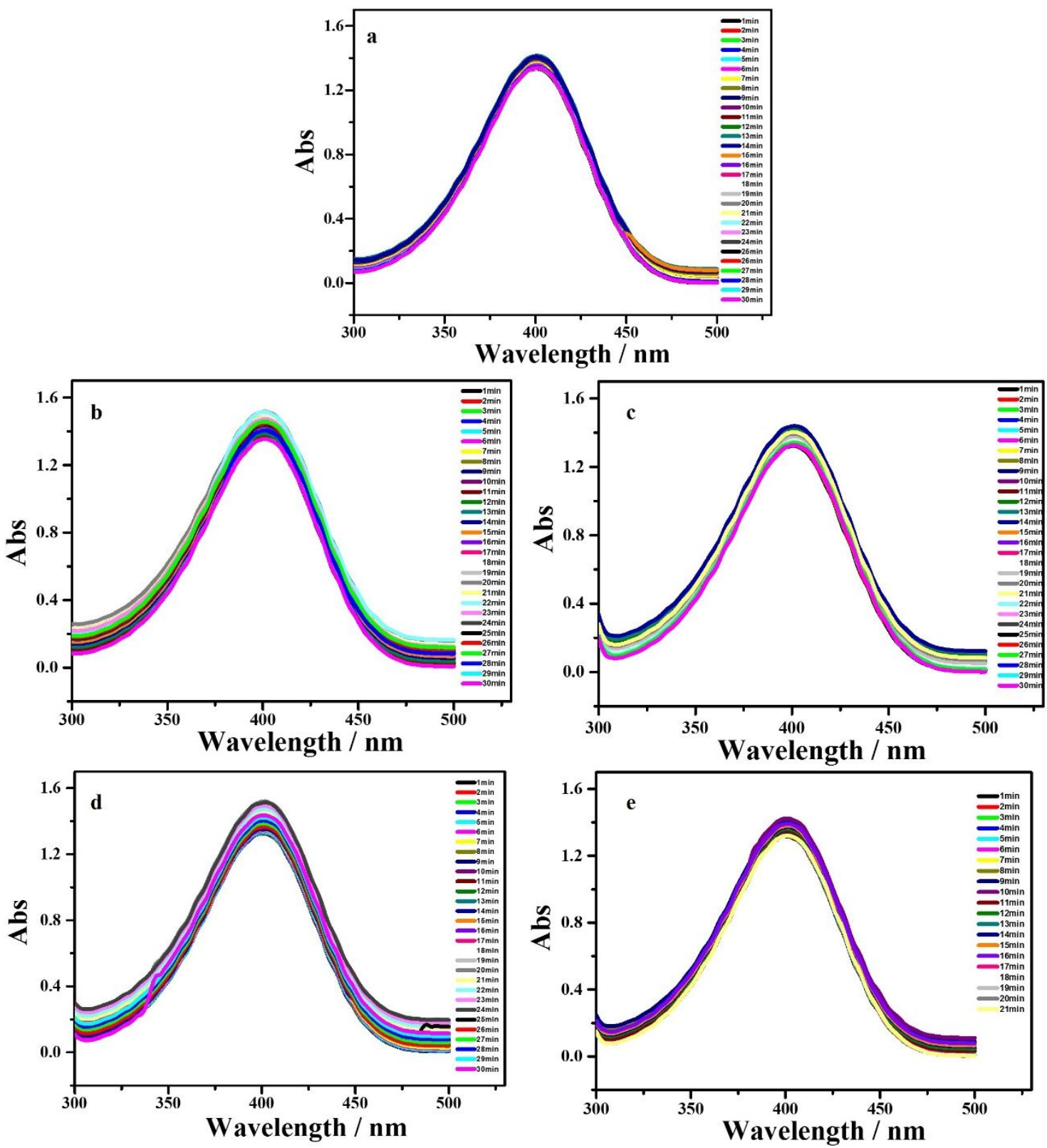

Figure S2. UV-vis spectra of the reduction of 4-NP by $\mathrm{NaBH}_{4}$ in presence of $\mathrm{H}_{2} \mathrm{O}$ (a), A (b), G (c), C(d), and T (e) without AuNRs at $298 \mathrm{~K}$, respectively. 


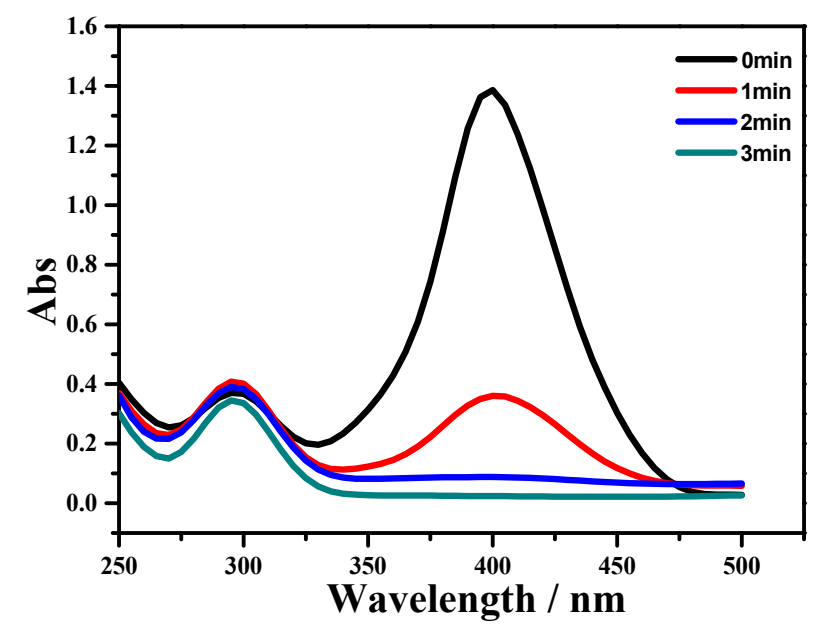

Figure S3. UV-vis spectra of 4-NP by $\mathrm{NaBH}_{4}$ in presence of AuNRs
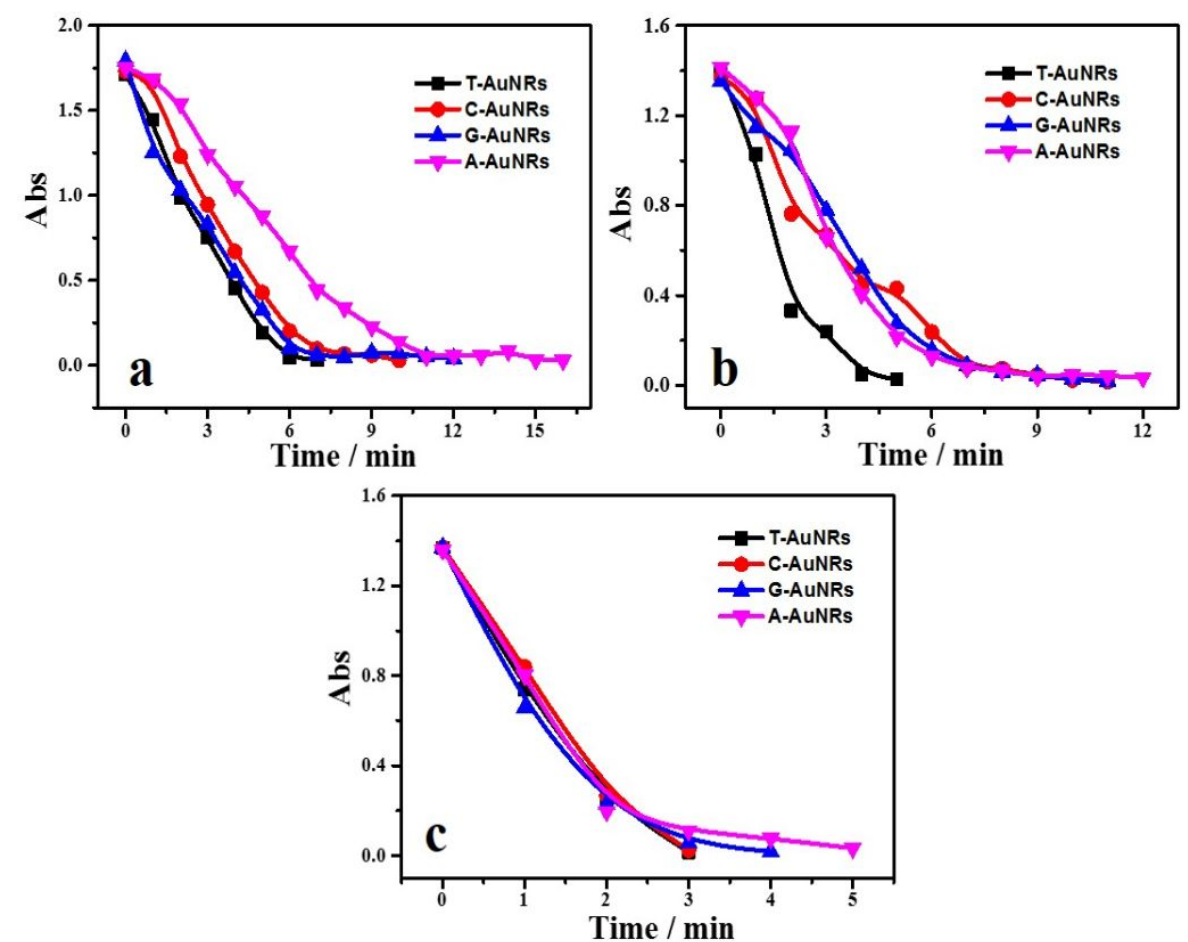

Figure S4. The absorption at $400 \mathrm{~nm}$ vs time of 4-NP reduction of AuNRs in the presence of different concentration of same nucleobases at $298 \mathrm{~K} .5 \mu \mathrm{L}$ of $15 \mathrm{mmol} \cdot \mathrm{L}^{-1}$ (a), $5.0 \mathrm{mmol} \cdot \mathrm{L}^{-1}$ (b), and $1.0 \mathrm{mmol} \cdot \mathrm{L}^{-1}$ (c) nucleobases were added to $45 \mu \mathrm{L}$ AuNRs solutions, respectively. 




Figure S5. The catalytic efficiencies of 4-NP reduction in presence of different catalysts at $298 \mathrm{~K}$.

Comparison investigation on the catalytic activity of nucleobases modified AuNRs at different temperatures by UV-vis spectra





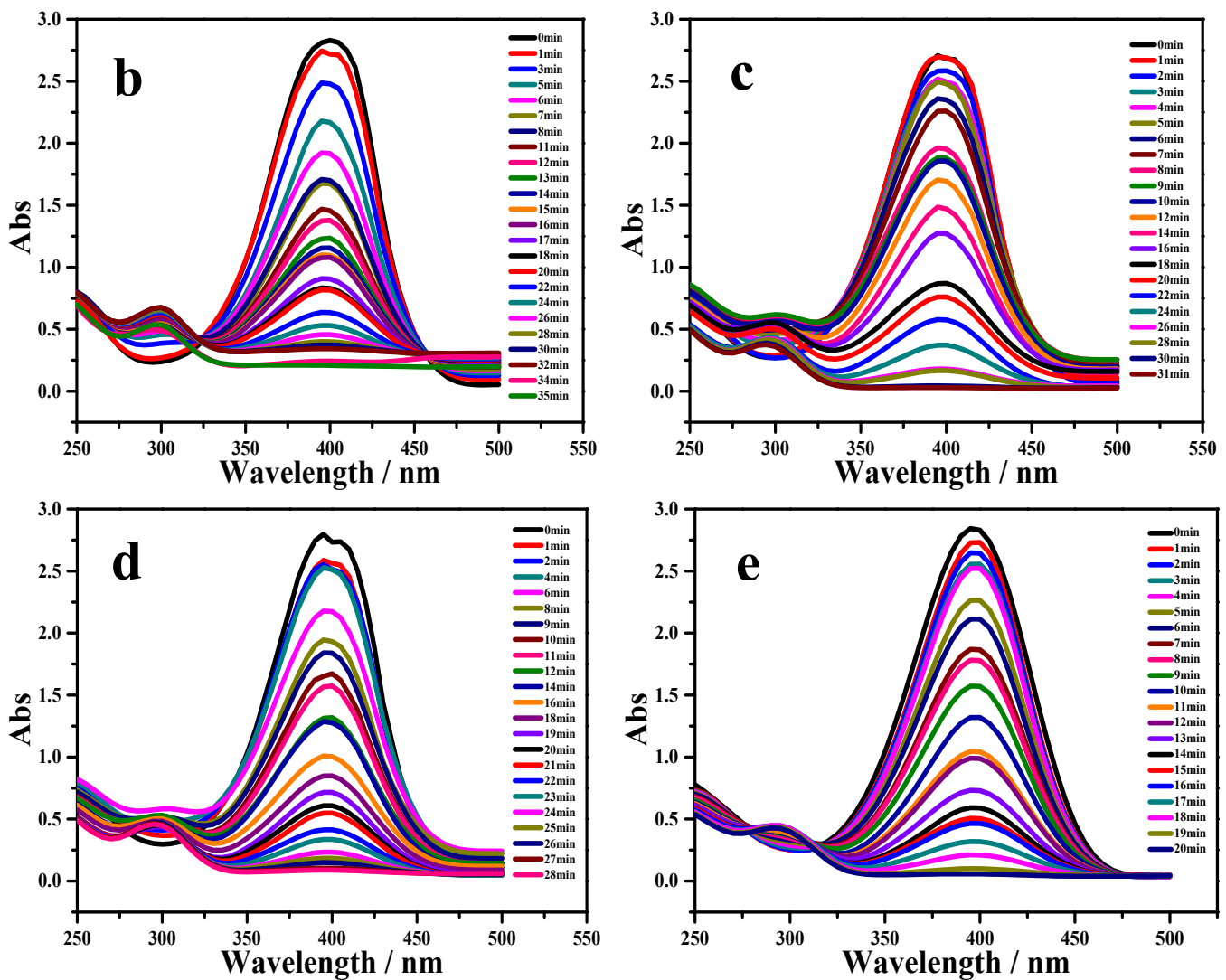

Figure S6. At $288 \mathrm{~K}, \mathrm{UV}$-vis spectra of 4-NP by $\mathrm{NaBH}_{4}$ in presence of AuNPs and nucleobases modified AuNRs, respectively, (a) AuNPs, (b) A-AuNRs, (c) G-AuNRs, (d) C-AuNRs, (e) T-

AuNRs.

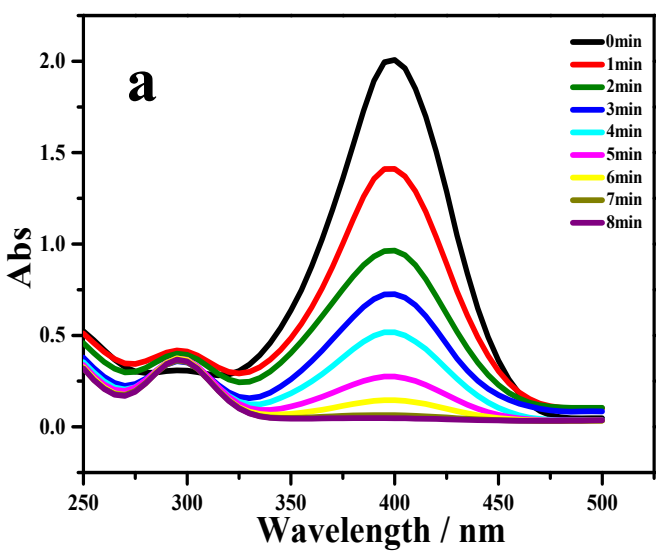



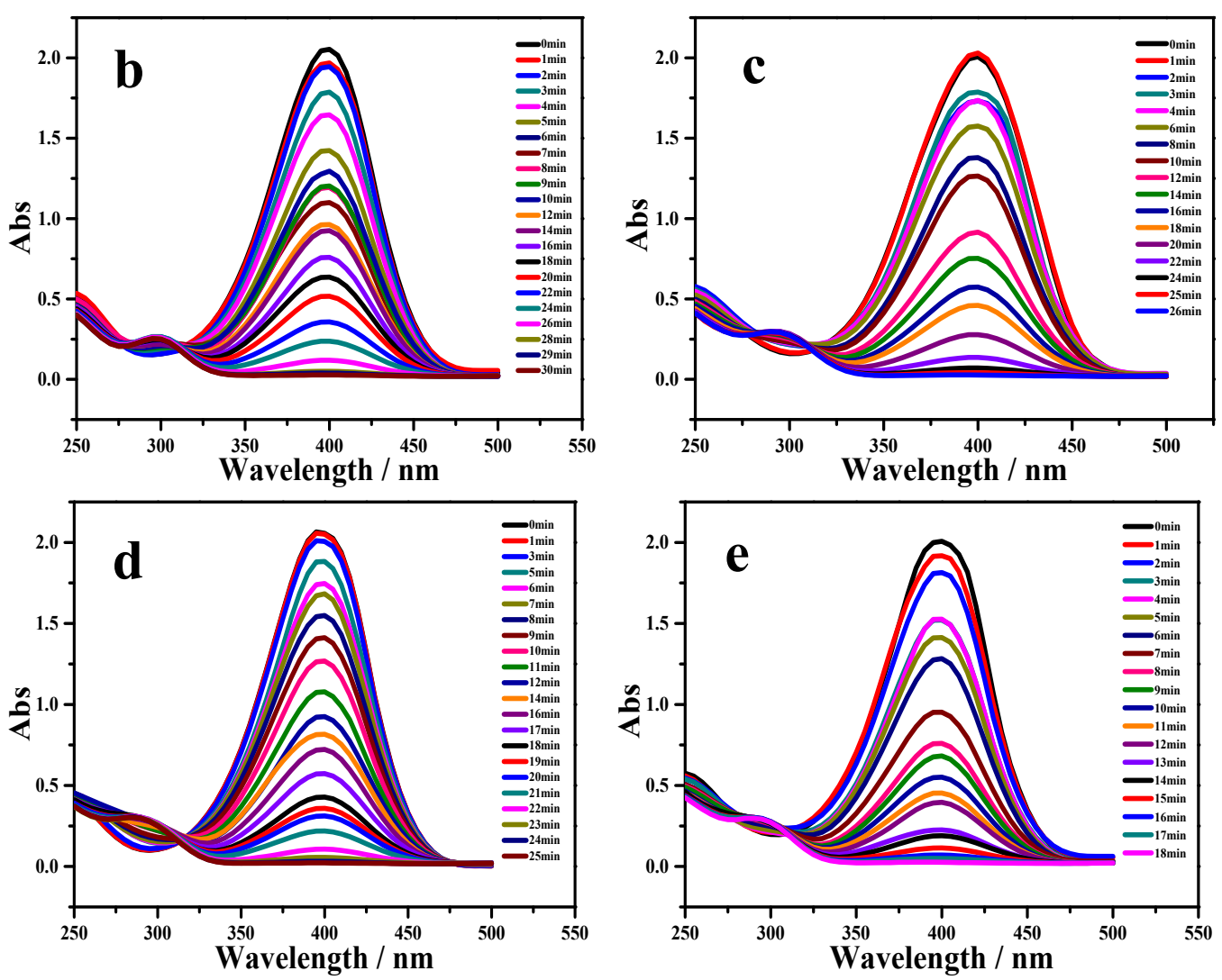

Figure S7. At $293 \mathrm{~K}, \mathrm{UV}$-vis spectra of 4-NP by $\mathrm{NaBH}_{4}$ in presence of AuNPs and nucleobases modified AuNRs, respectively, (a) AuNPs, (b) A-AuNRs, (c) G-AuNRs, (d) C-AuNRs, (e) T-

AuNRs.

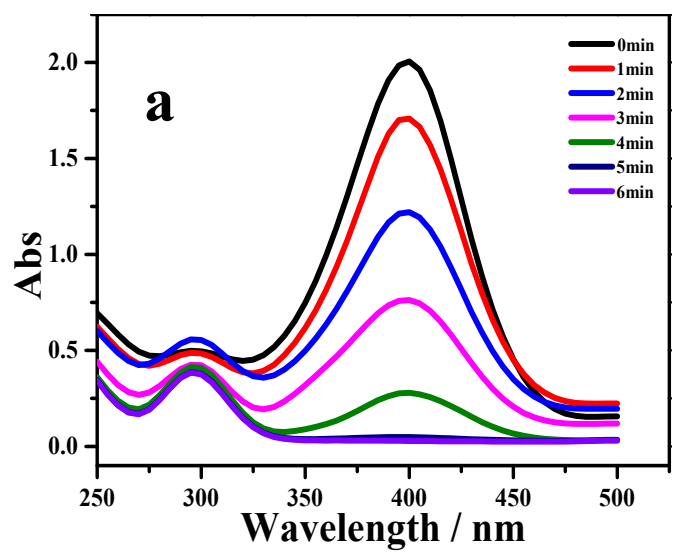



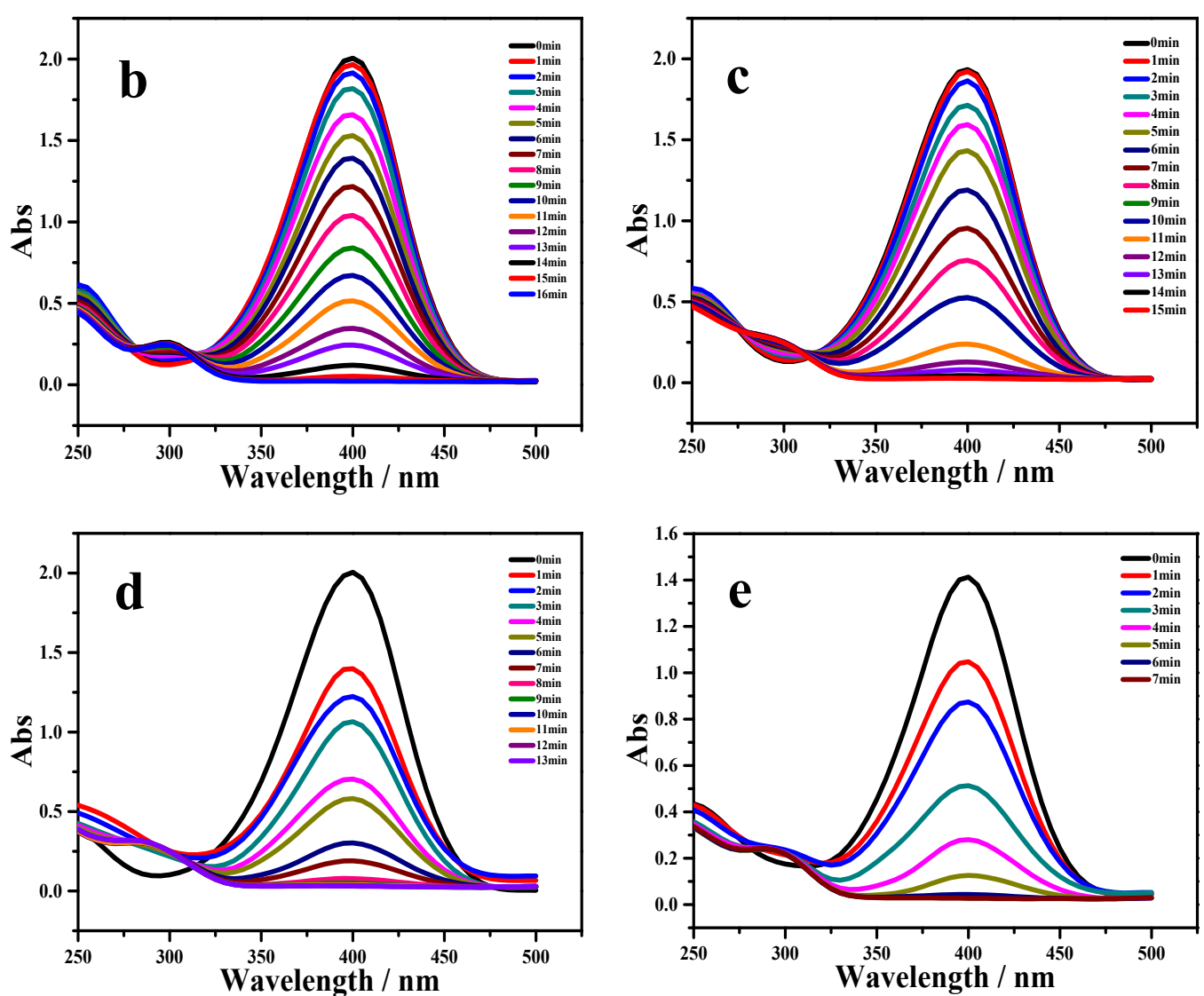

Figure S8. At 298 K,UV-vis spectra of 4-NP by $\mathrm{NaBH}_{4}$ in presence of AuNPs and nucleobases modified AuNRs, respectively, (a) AuNPs, (b) A-AuNRs, (c) G-AuNRs, (d) C-AuNRs, (e) TAuNRs.

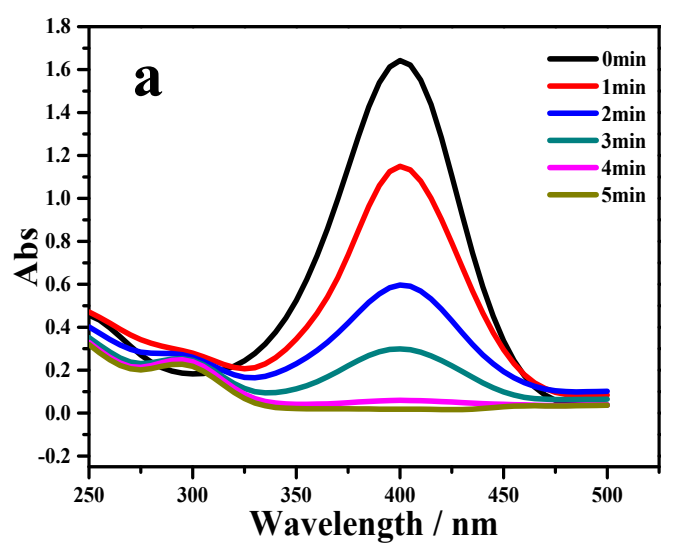



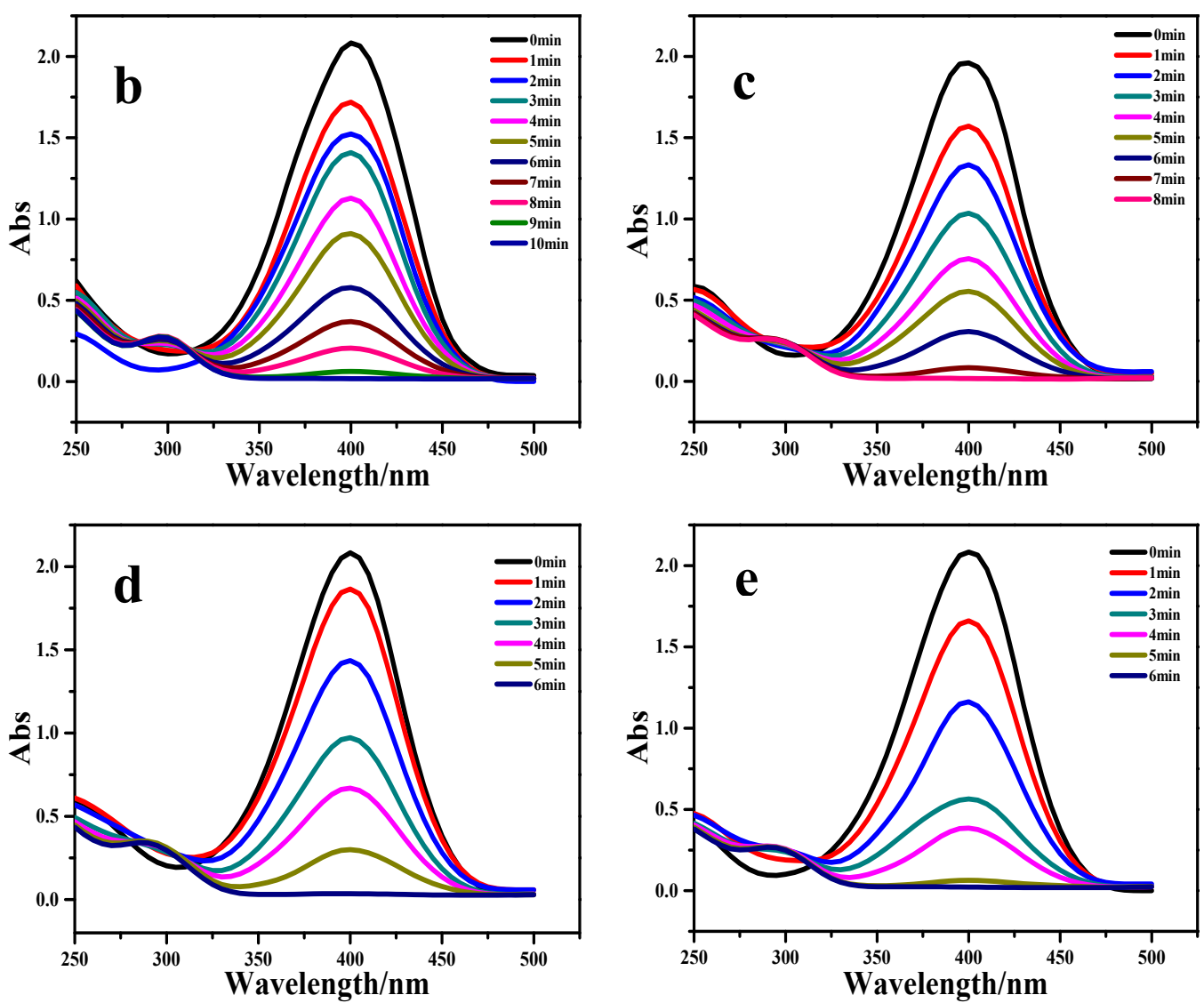

Figure S9. At $303 \mathrm{~K}, \mathrm{UV}$-vis spectra of 4-NP by $\mathrm{NaBH}_{4}$ in presence of AuNPs and nucleobases modified AuNRs, respectively, (a) AuNPs, (b) A-AuNRs, (c) G-AuNRs, (d) C-AuNRs, (e) TAuNRs.

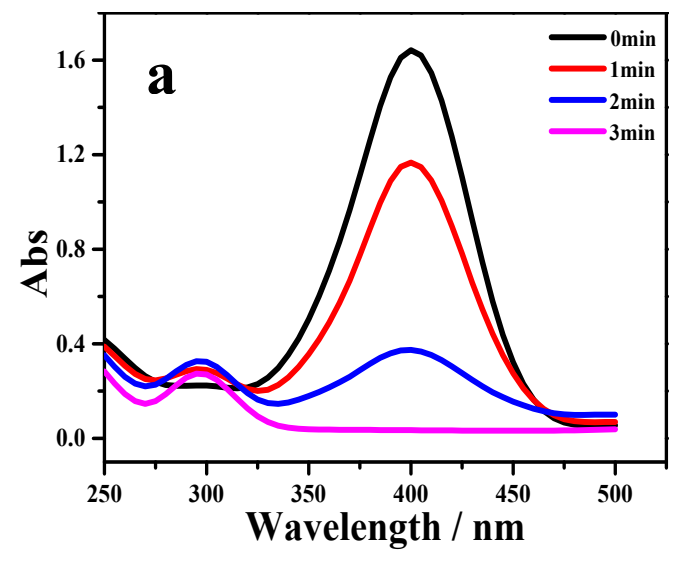



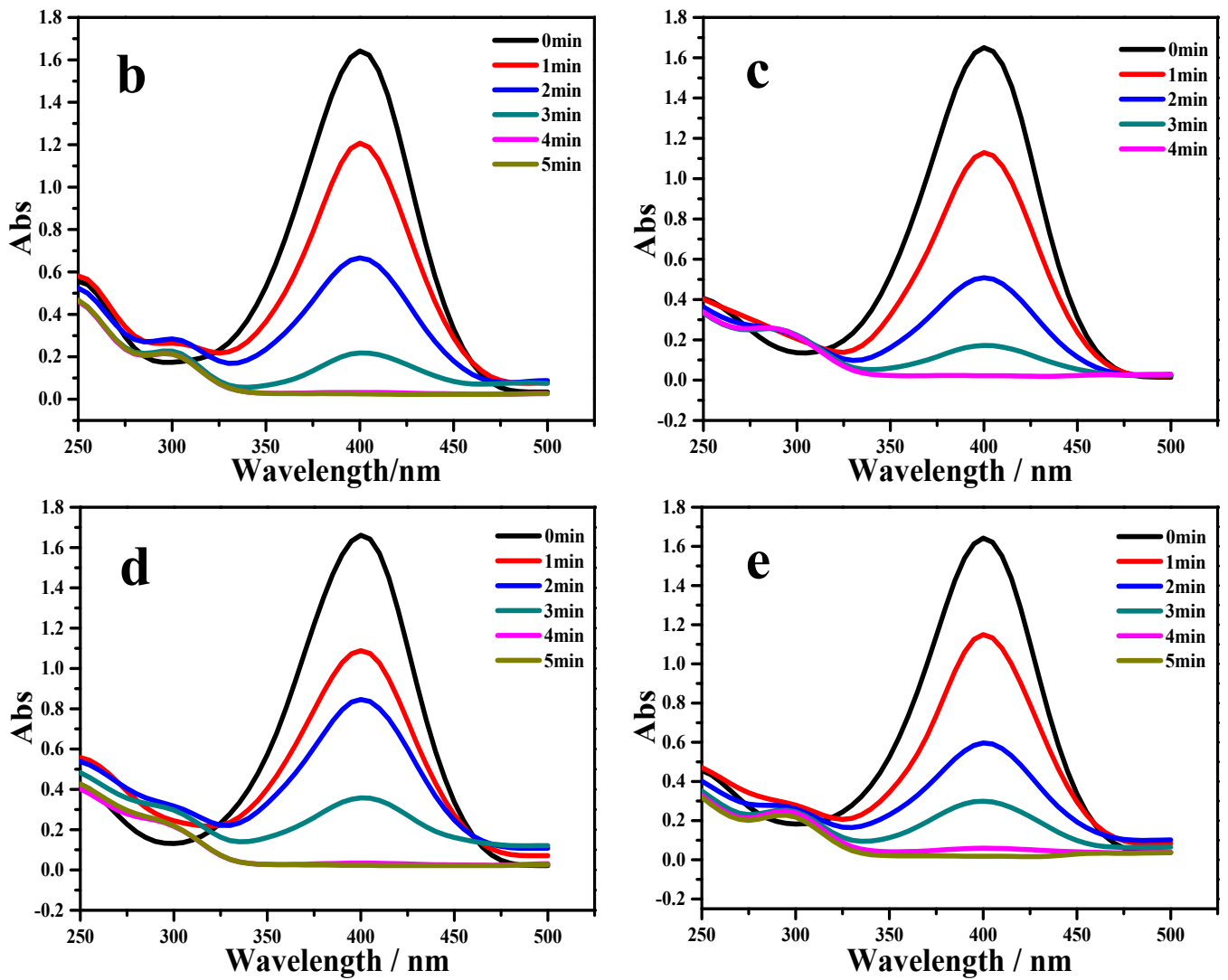

Figure S10. At 308 K,UV-vis spectra of 4-NP by $\mathrm{NaBH}_{4}$ in presence of AuNPs and nucleobases modified AuNRs, respectively, (a) AuNPs, (b) A-AuNRs, (c) G-AuNRs, (d) C-AuNRs, (e) TAuNRs. 

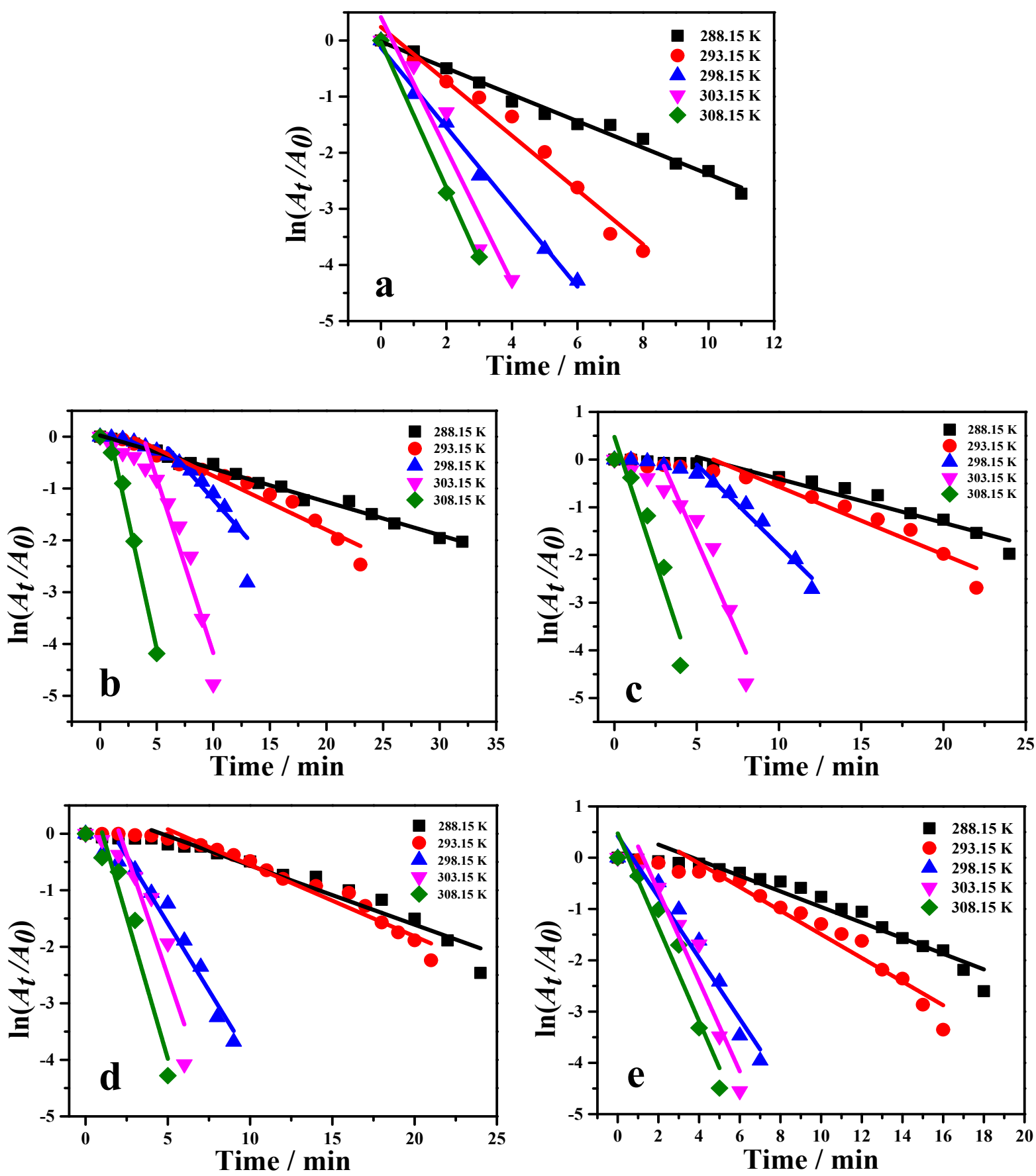

Figure S11. Plots of $\ln \left(A_{t} / A_{0}\right)$ vs time of 4-NP reduction in the presence of AuNPs and nucleobases modified AuNRs, respectively. (a) AuNPs, (b) A-AuNRs, (c) G-AuNRs, (d) CAuNRs, (e) T-AuNRs. 
Catalytic activity investigation on homo-oligonucleotides (different lengths and sequences) modified AuNPs, based on 4-NP reduction reaction
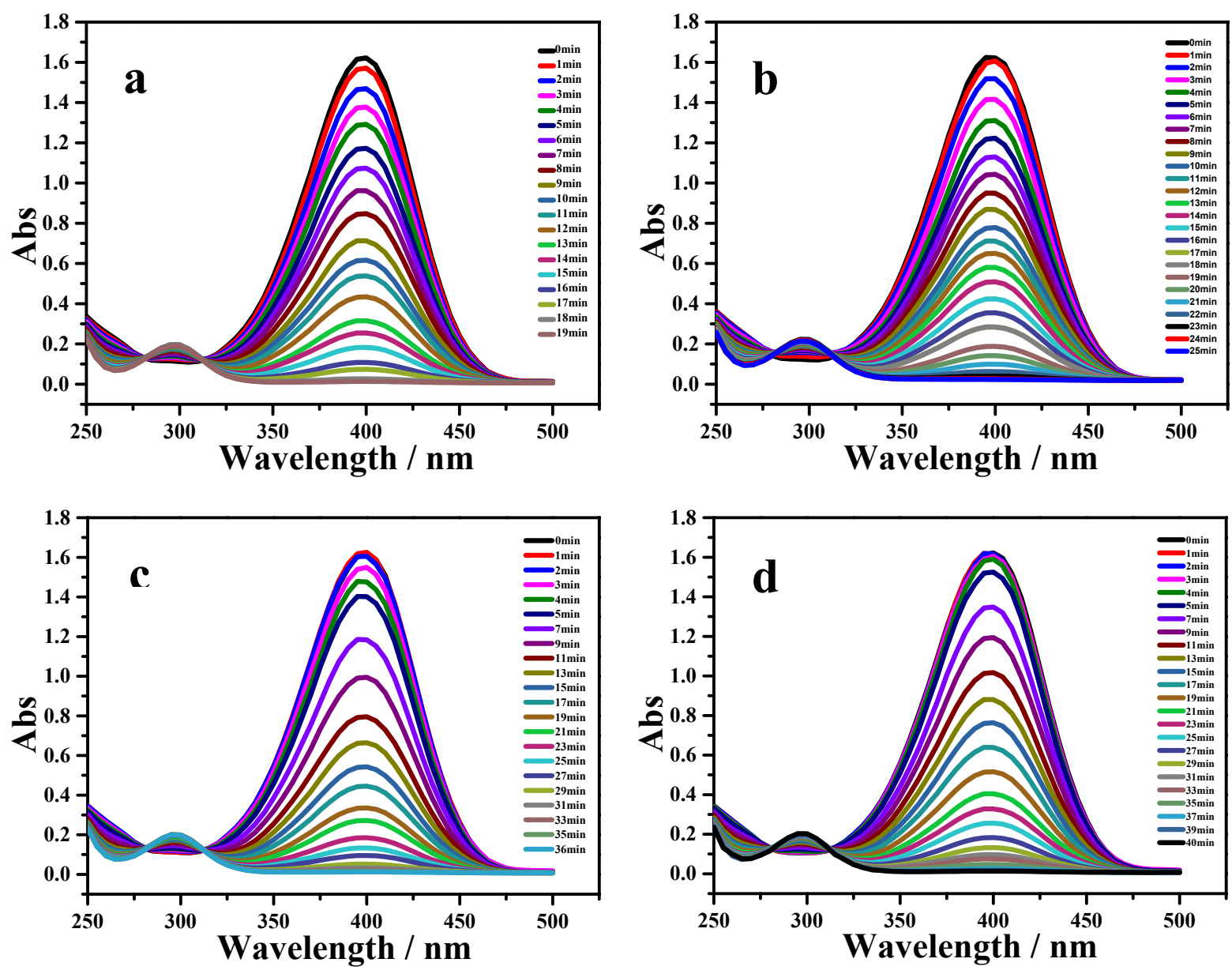

Figure S12. At $298 \mathrm{~K}, \mathrm{UV}$-vis spectra of 4-NP by $\mathrm{NaBH}_{4}$ in presence of homo-oligonucleotides (sequences of A) modified AuNPs, respectively, (a) A5-AuNPs, (b) A10-AuNRs, (c) A20-AuNRs, (d) A30-AuNRs.

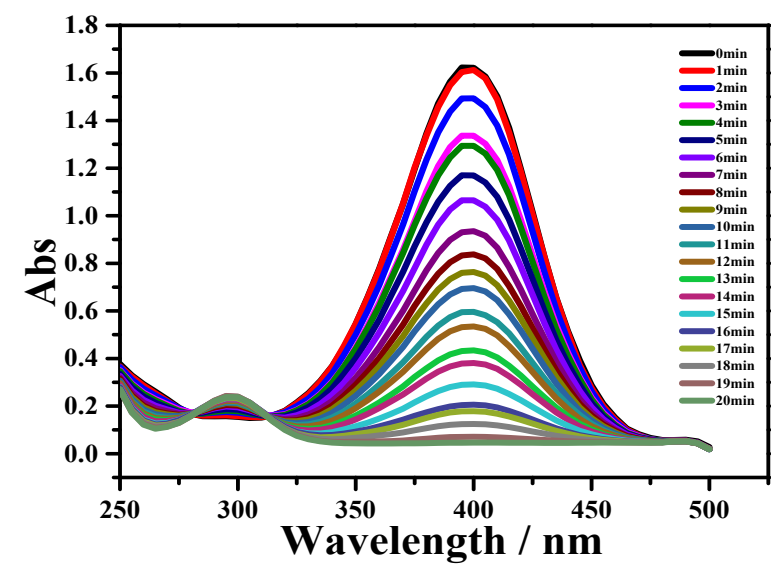

Figure S13. At $298 \mathrm{~K}, \mathrm{UV}$-vis spectra of 4-NP by $\mathrm{NaBH}_{4}$ in presence of G5-AuNRs 

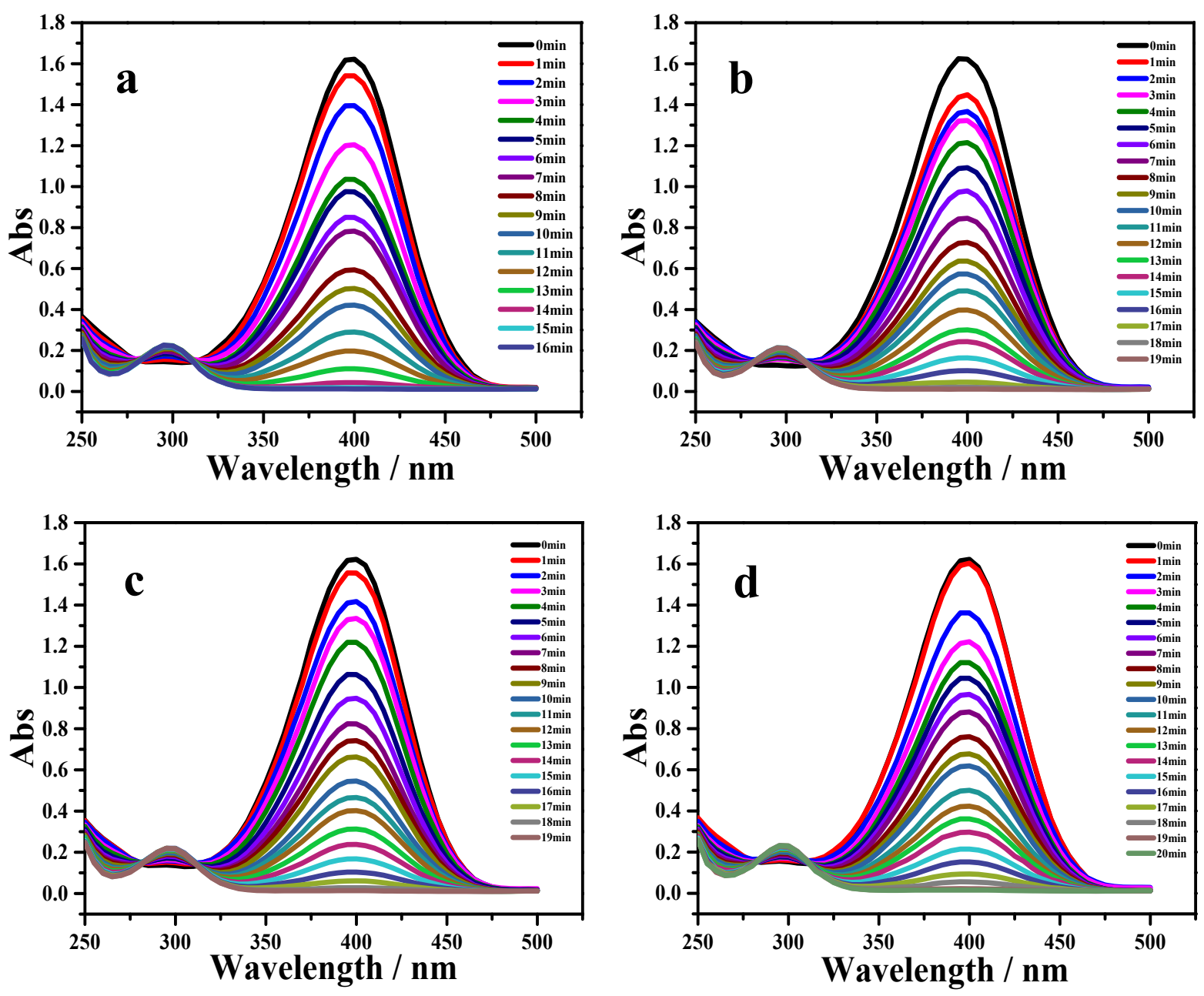

Figure S14. At 298 K,UV-vis spectra of 4-NP by $\mathrm{NaBH}_{4}$ in presence of homo-oligonucleotides (sequences of C) modified AuNPs, respectively, (a) C5-AuNPs, (b) C10-AuNRs, (c) C20-AuNRs, (d) C30-AuNRs.
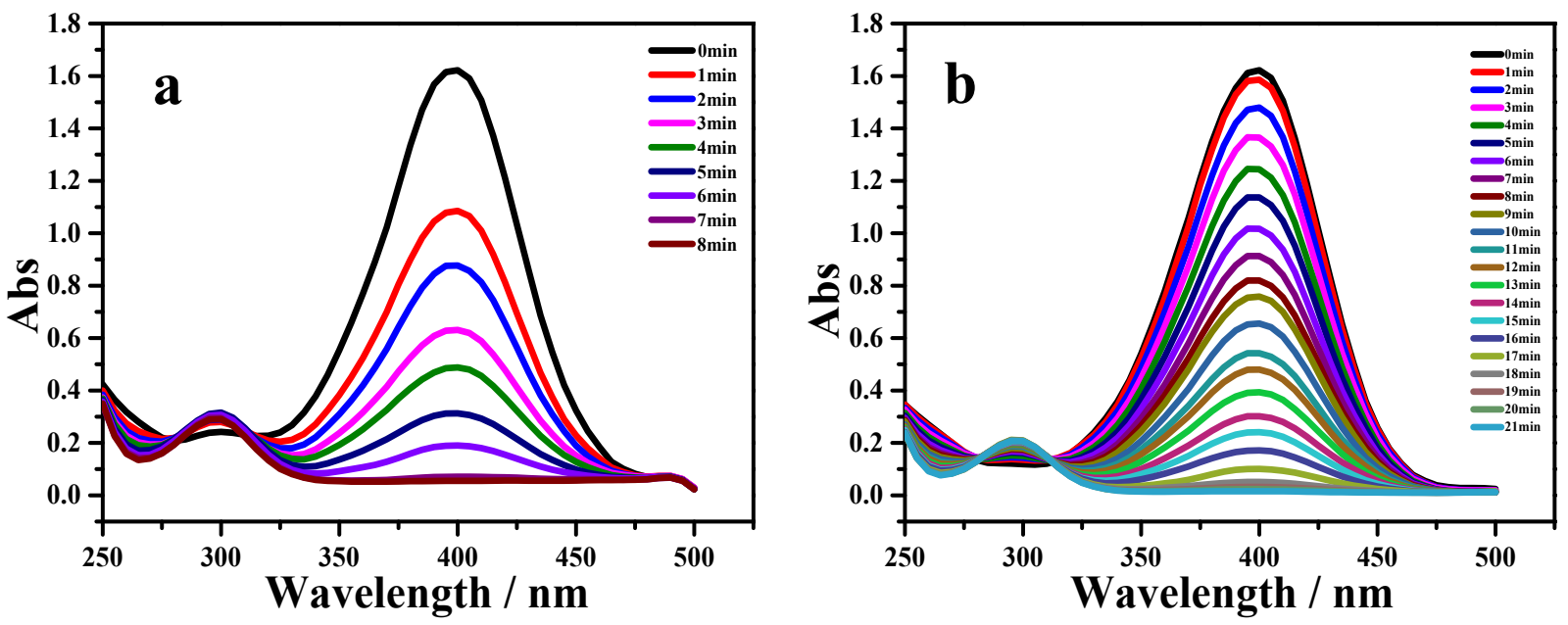

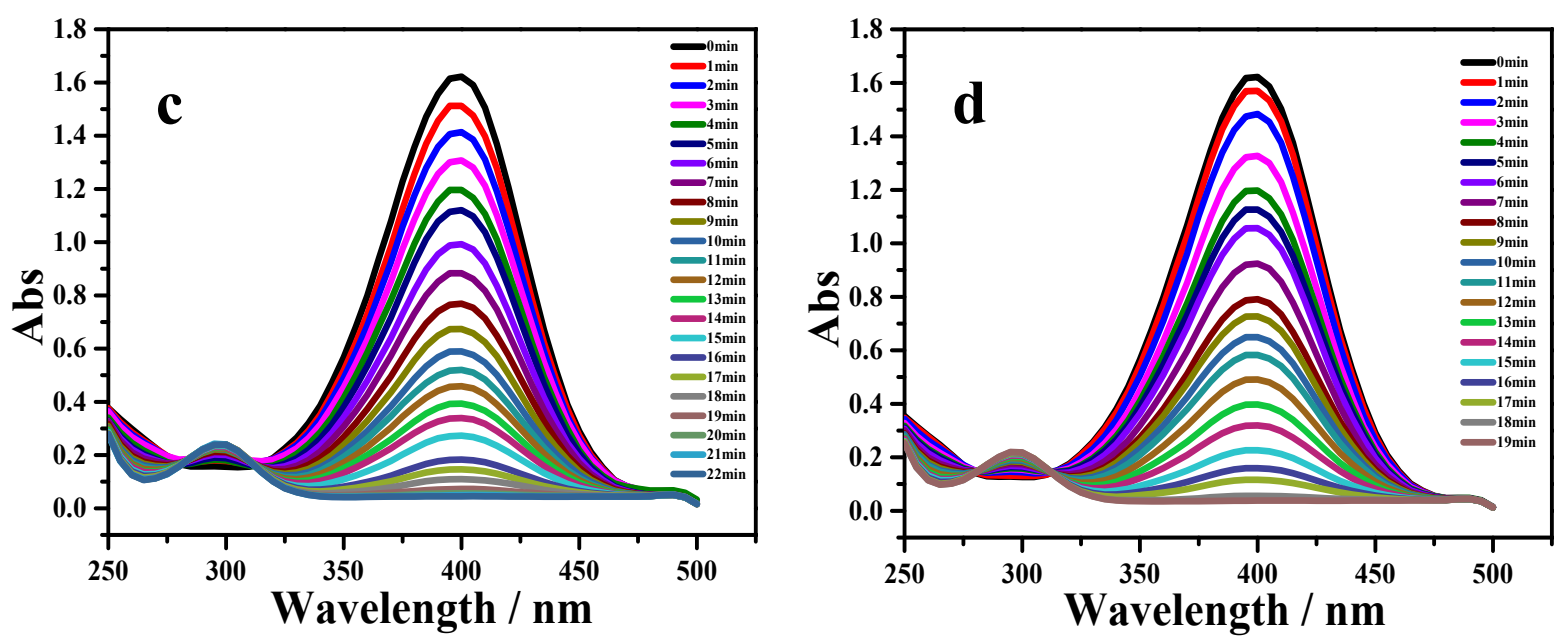

Figure S15. At 298 K,UV-vis spectra of 4-NP by $\mathrm{NaBH}_{4}$ in presence of homo-oligonucleotides (sequences of A) modified AuNPs, respectively, (a) T5-AuNPs, (b) T10-AuNRs, (c) T20-AuNRs, (d) T30-AuNRs. 

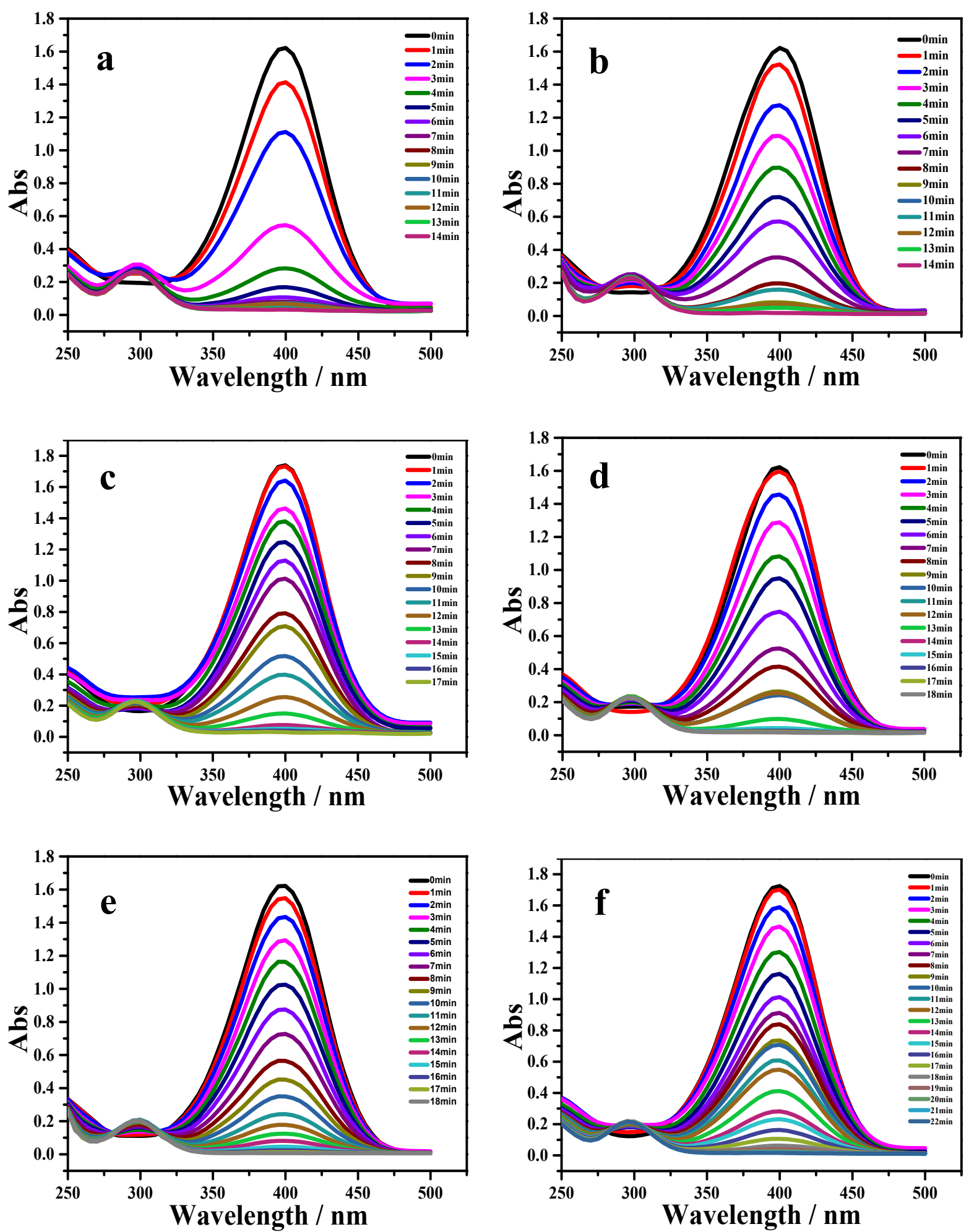

Figure S16. At $298 \mathrm{~K}$, UV-vis spectra of 4-NP by $\mathrm{NaBH}_{4}$ in presence of oligonucleotides-AuNRs (salt aging), respectively. (a) A-AuNRs, (b) A5-AuNRs, (c) A10-AuNRs, (d) A20-AuNRs, (e) A30-AuNRs, (f) A30+T30-AuNRs 

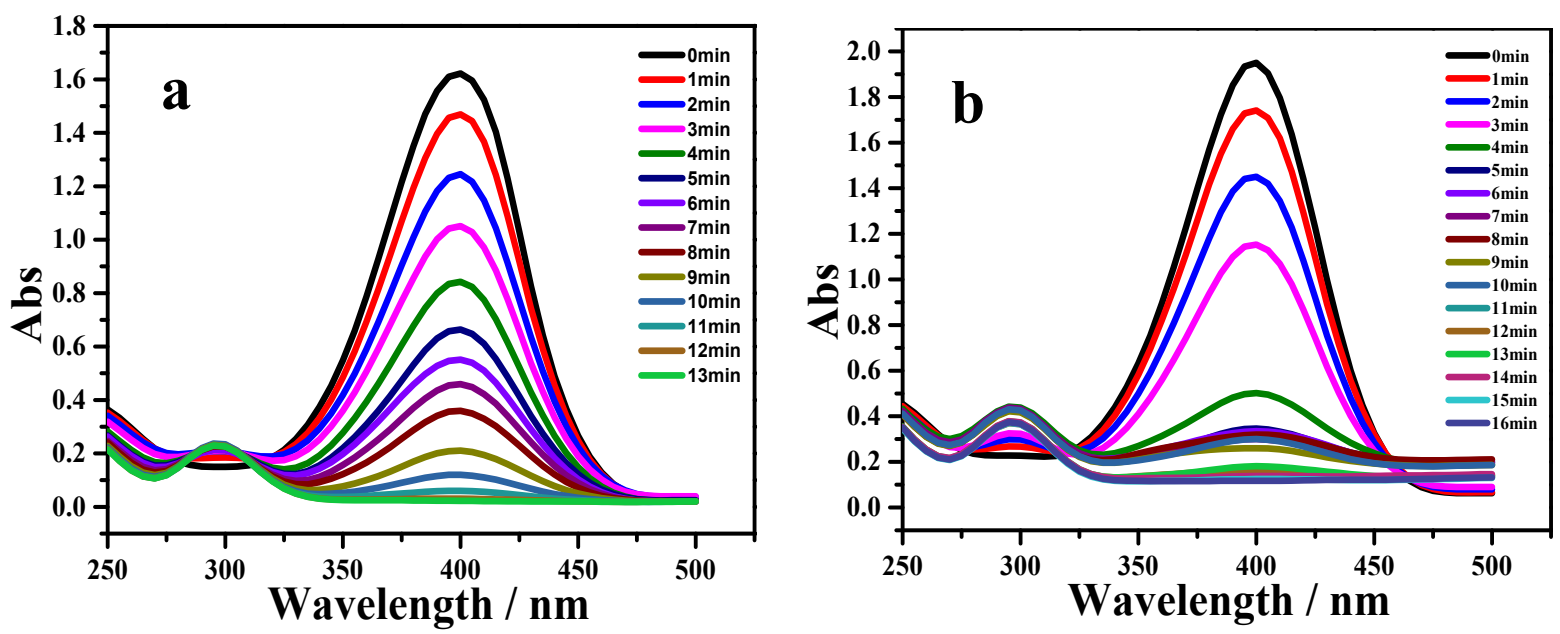

Figure S17. At $298 \mathrm{~K}$, UV-vis spectra of 4-NP by $\mathrm{NaBH}_{4}$ in presence of oligonucleotides-AuNRs (salt aging), respectively. (a) G-AuNRs, (b) G5-AuNRs. 

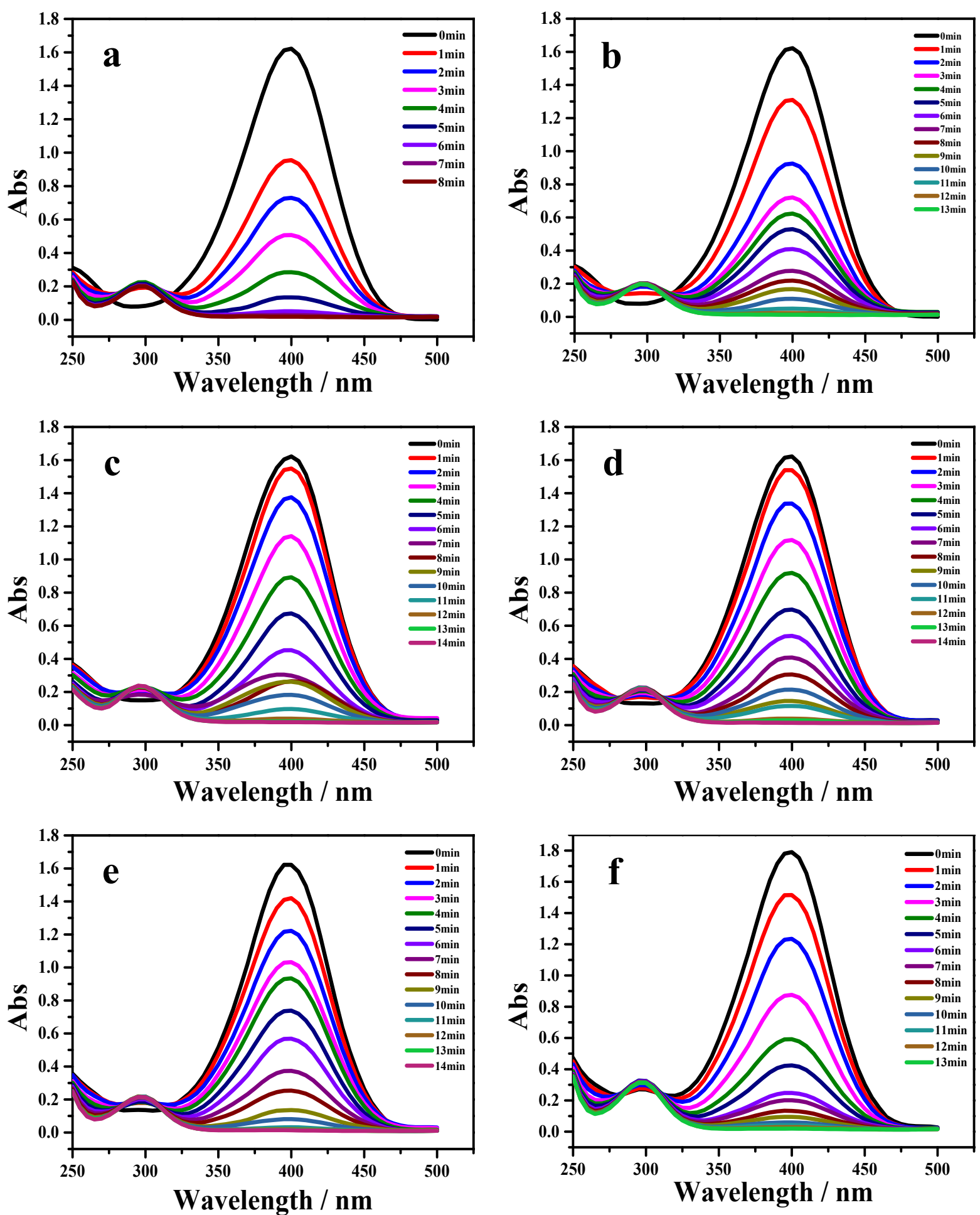

Figure S18. At $298 \mathrm{~K}$, UV-vis spectra of 4-NP by $\mathrm{NaBH}_{4}$ in presence of oligonucleotides-AuNRs (salt aging), respectively. (a) AuNRs, (b) C-AuNRs, (c) C5-AuNRs, (d) C10-AuNRs, (e) C20AuNRs, (f) C30-AuNRs 

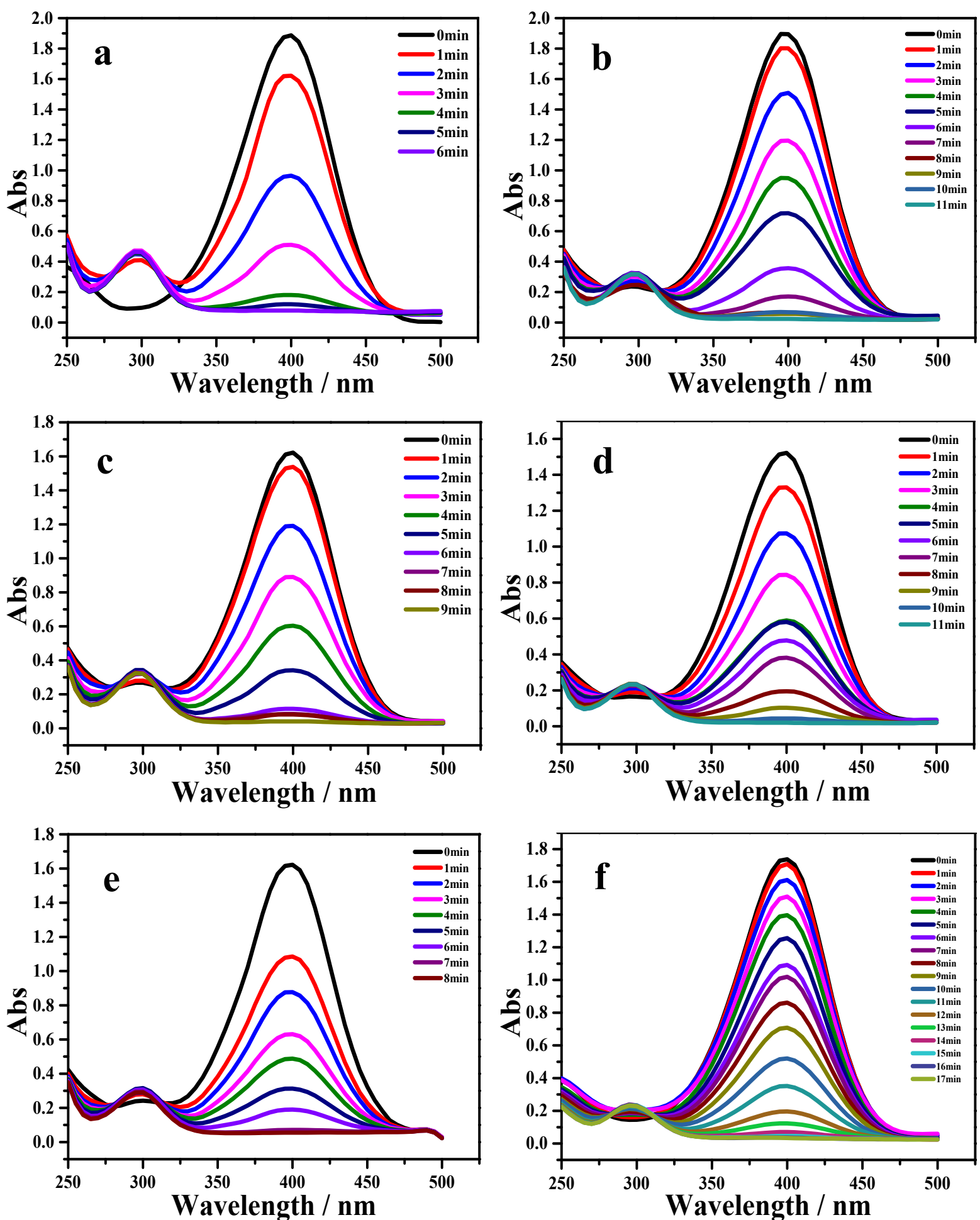

Figure S19. At $298 \mathrm{~K}$, UV-vis spectra of 4-NP by $\mathrm{NaBH}_{4}$ in presence of oligonucleotides-AuNRs

(salt aging), respectively. (a) T-AuNRs, (b) T5-AuNRs, (c) T10-AuNRs, (d) T20-AuNRs, (e) T30-

AuNRs, (f) T30+A30-AuNRs 
Table S1 Sequences of nucleobases and homo-oligonucleotides used in this work nucleobases and homosequences oligonucleotides names

\begin{tabular}{cl}
\hline adenine & $\mathrm{A}$ \\
thymine & $\mathrm{T}$ \\
cytosine & $\mathrm{G}$ \\
guanine & $\mathrm{C}$ \\
A5 & 5'-AAAAA-3' \\
A10 & 5'-AAAAAAAAAA -3' \\
A20 & 5'-AAAAAAAAAAAAAAA AAAAA -3' \\
A30 & 5'-AAAAAAAAAAAAAAAAAAAAAAAAAAAAAA -3' \\
G5 & 5'-GGGGG-3' \\
C5 & 5'-CCCCC-3' \\
C10 & 5'-CCCCCCCCCC-3' \\
C20 & 5'-CCCCCCCCCCCCCCCCCC-3' \\
C30 & 5'-CCCCCCCCCCCCCCCCCCCCCCCCCCC-3' \\
T5 & 5'-TTTTT-3' \\
T10 & 5'-TTTTTTTTT-3' \\
T20 & 5'-TTTTTTTTTTTTTTTTTT-3' \\
T30 & 5'-TTTTTTTTTTTTTTTTTTTTTTTTTTT-3' \\
\hline
\end{tabular}

Table S2 The reaction times $(t, \mathrm{~min})$ of 4-NP systems in presence of AuNRs and nucleobases (A/G/C/T) modified AuNRs at different temperatures, respectively.

\begin{tabular}{cccccc}
\hline$T / \mathrm{K}$ & AuNRs & A-AuNRs & G-AuNRs & C-AuNRs & T-AuNRs \\
\hline 288 & $18 \mathrm{~min}$ & $35 \mathrm{~min}$ & $31 \mathrm{~min}$ & $28 \mathrm{~min}$ & $20 \mathrm{~min}$ \\
293 & $8 \mathrm{~min}$ & $30 \mathrm{~min}$ & $26 \mathrm{~min}$ & $25 \mathrm{~min}$ & $18 \mathrm{~min}$ \\
298 & $6 \mathrm{~min}$ & $16 \mathrm{~min}$ & $15 \mathrm{~min}$ & $13 \mathrm{~min}$ & $7 \mathrm{~min}$ \\
303 & $4 \mathrm{~min}$ & $10 \mathrm{~min}$ & $8 \mathrm{~min}$ & $6 \mathrm{~min}$ & $6 \mathrm{~min}$ \\
308 & $3 \mathrm{~min}$ & $5 \mathrm{~min}$ & $5 \mathrm{~min}$ & $5 \mathrm{~min}$ & $4 \mathrm{~min}$ \\
\hline
\end{tabular}

